\title{
Faits et chiffres : La forte dispersion des revenus dans la viticulture française
}

\section{Bernard Delord}

\section{(2) OpenEdition}

\section{Journals}

\section{Édition électronique}

URL : http://journals.openedition.org/economierurale/3106

DOI : 10.4000/economierurale.3106

ISSN : 2105-2581

\section{Éditeur}

Société Française d'Économie Rurale (SFER)

\section{Édition imprimée}

Date de publication : 30 juillet 2011

Pagination : 60-70

ISSN : 0013-0559

\section{Référence électronique}

Bernard Delord, «Faits et chiffres : La forte dispersion des revenus dans la viticulture française », Économie rurale [En ligne], 324 | juillet-août 2011, mis en ligne le 30 juillet 2013, consulté le 19 avril 2019. URL : http://journals.openedition.org/economierurale/3106 ; DOI : 10.4000/ economierurale.3106 


\title{
La forte dispersion des revenus dans la viticulture française
}

\author{
Bernard DELORD • INRA, MOISA, Montpellier
}

Cette étude cherche à rendre compte des spécificités des revenus des viticulteurs, au regard de ceux des autres agriculteurs, quant à leur niveau, leur hétérogénéité, leur absence de lien apparent avec la productivité physique du travail et leur dépendance envers le niveau de prix de vente du vin (NDLR).

$\mathbf{E}_{\mathrm{n}}^{\mathrm{n}}$ dépit d'aides publiques importantes, le revenu moyen des agriculteurs français est voisin du SMIC, si on y inclut le coût de la protection sociale. Cependant, les différences de revenu entre exploitations sont fortes. Au sein de chaque système de production, ces différences sont en grande partie liées aux inégalités de productivité physique du travail, c'est-à-dire, généralement, aux disparités de dimension (Delord, 2005 ; Guyomard et al., 2007) ${ }^{1}$; toutefois, la relation entre le revenu et la dimension n'est pas linéaire (Butault, Delame, 2002) $)^{2}$.

La viticulture occupe, dans l'agriculture, une place particulière : le revenu moyen est nettement plus élevé, sa variabilité est plus importante et les aides publiques y jouent un rôle mineur. De plus, les exploitations viticoles sont plutôt de petite dimension

1. Delord B. (2005). Soutiens publics et emplois agricoles - Travail, emploi, productivité et aides publiques dans l'agriculture française en 2000. Montpellier, INRA, janvier.

Guyomard H., Chatellier V., Courleux F., Levert F. (2007). La politique de soutien des revenus agricoles dans l'UE : quel avenir pour les droits à paiement unique? In «Perspectives agricoles en France et en Europe », Paris, Rapport du Conseil d'analyse économique (CAE) du Premier ministre, p. 125-179. 2. Butault J.-P., Delame N. (2002). Croissance des exploitations et évolution des revenus agricoles : un mouvement brownien ? Une analyse à partir de l'échantillon constant RICA 91-97. INSEE Synthèses, ${ }^{\circ}$ 63, p. 53-64.
(Agreste, 2009) ${ }^{3}$, bien que croissante (Causeret et al., 1996), et la main-d'œuvre salariée y est importante (Traversac et al., $2007)^{5}$. Il faut sans doute rapprocher ce constat du passage de la production de vins indifférenciés à une production de vins majoritairement liés à des appellations, permettant une segmentation du marché. C'est un cas unique, du moins à l'échelle du pays entier ${ }^{6}$.

Cette étude vise à mesurer les différences de revenu au sein de la viticulture et de les rapprocher des caractéristiques des exploitations. Après avoir présenté le Réseau d'in-

3. Agreste (2009). Enquête sur la structure des exploitations agricoles en 2007. Chiffres et données Agriculture, $\mathrm{n}^{\circ}$ 201. Les résultats sont aussi disponibles sur : http://agreste.maapar.lbn.fr/ReportFolders/ReportFolders.aspx

4. Causeret F., Erguy T., Rio P. (1996). Évolution des exploitations viticoles en Languedoc-Roussillon : 1988-1995. Revue de l'Économie Méridionale, vol. 44, $\mathrm{n}^{\circ}$ 176, p. 11-32.

5. Traversac J.-B., Aubert M., Laporte J.-P., PerrierCornet Ph. (2007). Deux décennies d'évolution des structures de la viticulture française. In «Bacchus 2008 : enjeux, stratégies et pratiques dans la filière vitivinicole », Dunod.

6. Un autre cas, mais à une échelle plus réduite, est celui de la production laitière de la Savoie ou de Franche-Comté, adossée à des fromages $\mathrm{AOC}$ ou IGP. Chatellier V., Delattre F. (2003). La production laitière dans les montagnes françaises : une dynamique particulière pour les Alpes du Nord. INRA Production Animale, vol. $16, \mathrm{n}^{\circ} 1$, février, p. 61-76. 
formation comptable agricole (RICA), source sur laquelle s'appuie ce travail, on décrira la dispersion du revenu des viticulteurs et montrera la faiblesse des liens entre ce revenu et la productivité physique du travail. Enfin, en contrepoint, on cherchera l'origine des écarts de revenu dans la différenciation des prix unitaires de vente du vin.

\section{RICA et viticulture}

Le RICA est la seule enquête représentative nationale fournissant des données comptables sur les exploitations agricoles. Il convient cependant d'identifier les limites de son utilisation dans le champ de la viticulture.

\section{Le RICA}

Le RICA est une enquête comptable réalisée annuellement par le Service de la statistique et de la prospective (SSP, anciennement SCEES) sur un échantillon d'exploitations agricoles. Ayant pour objectif une représentation de l'agriculture professionnelle, ne figurent dans le RICA que des exploitations atteignant un seuil minimum de Marge brute standard (MBS) et d'activité. Ainsi, sont exclues de son champ, et donc du présent travail, les (très) petites exploitations, notamment celles relevant de l'agriculture à temps partiel, assez fréquentes en viticulture?. L'échantillon du RICA est stratifié par région d'Orientation technicoéconomique (OTEX) et classe de MBS. Chaque exploitation enquêtée est dotée d'un coefficient de pondération.

Pour tenir compte des fortes variations de récolte en viticulture, nous avons choisi de nous appuyer sur une moyenne trisannuelle. En fait, nous avons utilisé la méthode de l'échantillon constant du RICA 2005 à

7. Selon l'Enquête structure, en 2005 et en 2007, au sein des deux OTEX viticoles, les non-professionnelles représentent environ $36 \%$ du nombre d'exploitations, mais moins de $6 \%$ de la surface en vigne et $4 \%$ de la MBS (Agreste, 2009).
$2007^{8}$, qui repose sur l'identification des exploitations enquêtées présentes ${ }^{9}$ dans le RICA aux trois dates.

\section{Les exploitations viticoles dans le RICA}

S'agissant d'étudier les exploitations viticoles, nous avons retenu uniquement les exploitations enquêtées qui, aux trois dates, relèvent d'une OTEX viticole, « viticulture de qualité » ou « autre viticulture », à l'exception de celles spécialisées en vigne à raisin de table ${ }^{10}$.

De nouveaux coefficients de pondération ont été calculés, selon la même stratification que le RICA, pour que le nouvel échantillon reste représentatif des exploitations vinicoles de l'univers représenté par le RICA en $2007^{11}$.

Au sein de la viticulture, la vente en bouteille est un important facteur de différenciation (Traversac et al., op. cit. $)^{12}$. Ici, nous définissons comme "embouteilleurs », les exploitations dont les ventes (ou la production) de vin ou d'eau-de-vie en bouteille dépassent soit $75 \mathrm{hl}$ ou $30 \mathrm{k} €$, soit la moitié (en volume ou en valeur) des ventes (ou production) de vin et autres produits viticoles. On distingue donc les exploitations toujours (aux trois dates) à dominante bouteille, celles qui ne le sont jamais et celles qui sont dans un autre cas.

Selon cette définition, un peu plus du tiers des exploitations viticoles représentées (essentiellement dans l'OTEX AOC) sont considérées comme « embouteilleuses » (tableau 1). Les autres vendent leur vin quasi uniquement en vrac, ou vendent le

8. Il s'agit là des trois dernières années pour lesquelles nous avons pu accéder à l'ensemble des données du RICA.

9. Nous avons assimilé un coefficient de pondération inférieur à 1 à une absence.

10. C'est-à-dire celles dont la MBS des vignes à vin atteint ou dépasse les $2 / 3$ de la MBS totale.

11. Dans cette projection, trois strates non vides du RICA 2007 sont vides dans l'échantillon constant et, de ce fait, il manque environ 197 exploitations dans la représentation de l'univers.

12. Ibid., note 5 . 
Tableau 1. Caractéristiques des exploitations viticoles dans l'échantillon constant du RICA (2005/2006/2007)

\begin{tabular}{|c|c|c|c|c|c|c|c|c|}
\hline & \multirow[b]{2}{*}{$\begin{array}{l}\text { Nombre } \\
\text { de ques- } \\
\text { tionnaires }\end{array}$} & \multirow{2}{*}{$\begin{array}{l}\text { Nombre } \\
\text { d'exploi- } \\
\text { tations } \\
\text { repré- } \\
\text { sentées }\end{array}$} & \multicolumn{4}{|c|}{ Caractéristiques moyennes par exploitation } & \multicolumn{2}{|c|}{$\%$ d'exploitations... } \\
\hline & & & $\begin{array}{c}\text { MBS } \\
\text { (en k€) }\end{array}$ & $\begin{array}{l}\text { Main } \\
\text { d'œuvre } \\
\text { totale } \\
\text { (en uta) }\end{array}$ & $\begin{array}{c}\text { Main } \\
\text { d'œuvre } \\
\text { familiale } \\
\text { (en uta) }\end{array}$ & \begin{tabular}{|c|} 
Surface \\
en vignes \\
productives \\
(en ha)
\end{tabular} & $\begin{array}{l}\text {.... } \\
\text { embou- } \\
\text { teilleuses }\end{array}$ & $\begin{array}{c}\text {... } \\
\text { de l'otex } \\
\text { « qualité » }\end{array}$ \\
\hline $\begin{array}{l}\text { Ensemble } \\
\text { des OTEX }\end{array}$ & 5023 & 313060 & 82 & 2,0 & 1,5 & 3 & 5 & 10 \\
\hline $\begin{array}{l}\text { OTEX non- } \\
\text { vinicoles }\end{array}$ & 4226 & 270662 & 77 & 1,9 & 1,5 & 0 & 0 & 0 \\
\hline $\begin{array}{l}\text { OTEX « vin } \\
\text { qualité » }\end{array}$ & 621 & 32376 & 129 & 30 & 14 & 14 & 46 & 100 \\
\hline $\begin{array}{l}\text { OTEX } \\
\text { " autre }\end{array}$ & & & & & & & & \\
\hline vin $"$ & 176 & 10022 & 68 & 2,0 & 1,2 & 23 & 4 & 0 \\
\hline $\begin{array}{l}\text { Total } \\
\text { vinicole }\end{array}$ & 797 & 42398 & 115 & 2,8 & 1,4 & 16 & 182 & 36 \\
\hline
\end{tabular}

Mise en bouteille :

\begin{tabular}{l|r|r|r|r|r|r|r|r} 
toujours & 320 & 15322 & 160 & 4,3 & 1,6 & 15 & 100 & 97 \\
jamais-vcc & 158 & 9349 & 66 & 1,8 & 1,2 & 22 & 0 & 0 \\
jamais-aoc & 272 & 14511 & 105 & 2,0 & 1,3 & 15 & 0 & 100 \\
autre cas & 47 & 3216 & 85 & 2,3 & 1,3 & 12 & 0 & 92 \\
\hline
\end{tabular}

Zones viticoles:

\begin{tabular}{l|r|r|r|r|r|r|r|r} 
Champ. & 68 & 6605 & 141 & 3,1 & 1,2 & 4 & 67 & 100 \\
Val-Loire & 50 & 2361 & 110 & 3,0 & 1,4 & 21 & 48 & 100 \\
Bourg. & 71 & 2959 & 169 & 3,5 & 1,6 & 11 & 62 & 100 \\
Alsace & 50 & 2102 & 116 & 2,9 & 1,6 & 7 & 54 & 100 \\
Poit.-Ch. & 40 & 2673 & 99 & 2,1 & 1,4 & 22 & 0 & 1 \\
Aqu.-M. Pyr. & 163 & 8135 & 180 & 3,3 & 1,5 & 18 & 44 & 97 \\
Rh.-Alp. & 70 & 3288 & 87 & 3,0 & 1,5 & 13 & 40 & 96 \\
Lang.-Rous. & 171 & 10243 & 59 & 2,0 & 1,1 & 24 & 9 & 38 \\
PACA & 88 & 3847 & 77 & 2,9 & 1,5 & 20 & 25 & 86 \\
Corse & 26 & 185 & 83 & 3,3 & 1,2 & 22 & 40 & 57 \\
\hline
\end{tabular}

Source : RICA-échantillon constant-2005/2006/2007, traitement INRA-MOISA

raisin en l'état, ou bien livrent leur raisin à une coopérative.

Il aurait été utile de distinguer les viticulteurs coopérateurs des viticulteurs vinifiant leur raisin et vendant du vin en vrac. Mais, dans le RICA, il n'existe aucun moyen, ni direct, ni indirect, de les différencier.

Ne disposant pas de précisions géographiques adaptées à la délimitation des zones d'appellation, nous avons dû nous limiter à un découpage selon les régions administratives en regroupant celles ayant des productions similaires : Champagne-Ardenne avec Île-de-France et Picardie, Bourgogne avec Franche-Comté, Pays de la Loire avec
Centre et Auvergne, Aquitaine avec MidiPyrénées (tableau 1).

\section{Les variables de l'analyse}

Nous cherchons ici à mesurer la valeur ajoutée ${ }^{13}$ créée sur l'exploitation, plus précisément à en préciser la formation et la répartition. Pour cela, on utilise la valeur ajoutée nette : (production de l'exercice augmentée des subventions d'exploitation et diminuée des approvisionnements et services acquis à l'extérieur (consommations intermédiaires)) et de l'amortissement des immobilisations (non-foncières et non-financières) utilisées par l'exploitation. On est là dans l'optique de la production. 
Du point de vue de la répartition, la valeur ajoutée nette permet d'acquitter les charges financières (nettes des revenus financiers), les impôts et taxes sur la production, et la rente foncière. Après les prélèvements par l'extérieur, le solde rémunère le travail (salarié et familial) ; ce solde est ici qualifié de « revenu total », c'est la partie de la valeur ajoutée qui reste sur l'exploitation. Le revenu des salariés inclut la protection sociale (charges patronales); le revenu familial est quant à lui égal au résultat courant avant impôts (RCAI) ${ }^{14}$. Considérant la productivité du travail et le partage de la valeur, il est plus logique de s'appuyer sur la valeur ajoutée nette et le RCAI que sur le RBE (ou sur le revenu disponible) pour ne pas écarter le travail salarié, tout en déduisant la charge du renouvellement des immobilisations, même si celui-ci n'est pas effectué régulièrement chaque année. Revenu total, revenu familial comme valeur ajoutée nette seront souvent considérés unitairement, c'est-à-dire, ramenés à l'unité de travail annuel (UTA).

Le montant de la production de l'exercice correspond à la somme des ventes de produits (viticoles et non viticoles), des variations de stocks, de l'autoconsommation et des produits annexes. Pour les viticulteurs coopérateurs, les ventes de vin correspondent aux acomptes perçus de la coopérative, les frais de vinification étant déduits ${ }^{15}$.

Le prix unitaire de vente du vin est le quotient du montant des ventes de ce produit (en vrac ou en bouteille) par le volume vendu. En l'absence de telle vente, le prix de vente n'est pas calculé.

13. Au sens commun du terme, i.e. au prix vendu par l'exploitation.

14. L'amortissement du capital et les charges financières ont été déduits ; mais ne le sont ni le remboursement en capital des emprunts, ni les cotisations sociales personnelles de l'exploitant et des autres non-salariés.

15. Pour ces viticulteurs coopérateurs, le prix unitaire de vente du vin est assez conventionnel puisqu'il résulte d'un calcul fondé sur les estimations des comptables-enquêteurs; de plus, à la différence des non-coopérateurs, il est net des frais de vinification.

\section{Schéma simplifié de définition des variables de revenu}

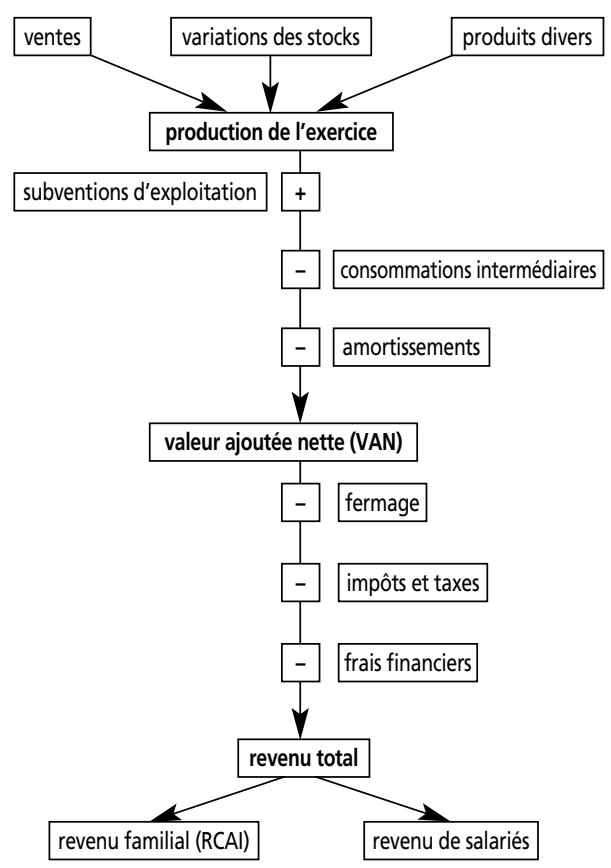

\section{Revenu et productivité du travail}

Dans cette partie, nous montrerons les différences importantes de revenu entre les viticulteurs et nous chercherons à les mettre en relation avec les facteurs que l'on sait bien corrélés avec le revenu des agriculteurs des autres orientations.

\section{Des niveaux de revenu très dispersés}

En viticulture, le revenu familial (RCAI) unitaire (par travailleur familial) moyen est élevé : il dépasse de moitié celui de l'ensemble de l'agriculture française (SCEES, $2007)^{16}$. Mais, encore plus que dans celle-ci, les revenus individuels sont très dispersés.

Pour la moitié des exploitations de l'OTEX « viticulture de qualité », comme pour l'ensemble de l'agriculture française, le revenu familial par équivalent temps

16. SCEES (2007). RICA France - Tableaux standard 2005. Agreste, Chiffres et Données, Série Agriculture, $\mathrm{n}^{\circ} 189$, mars, aussi disponible sur: http://agreste.maapar.lbn.fr/TableViewer/tableView.aspx?ReportId=6368 


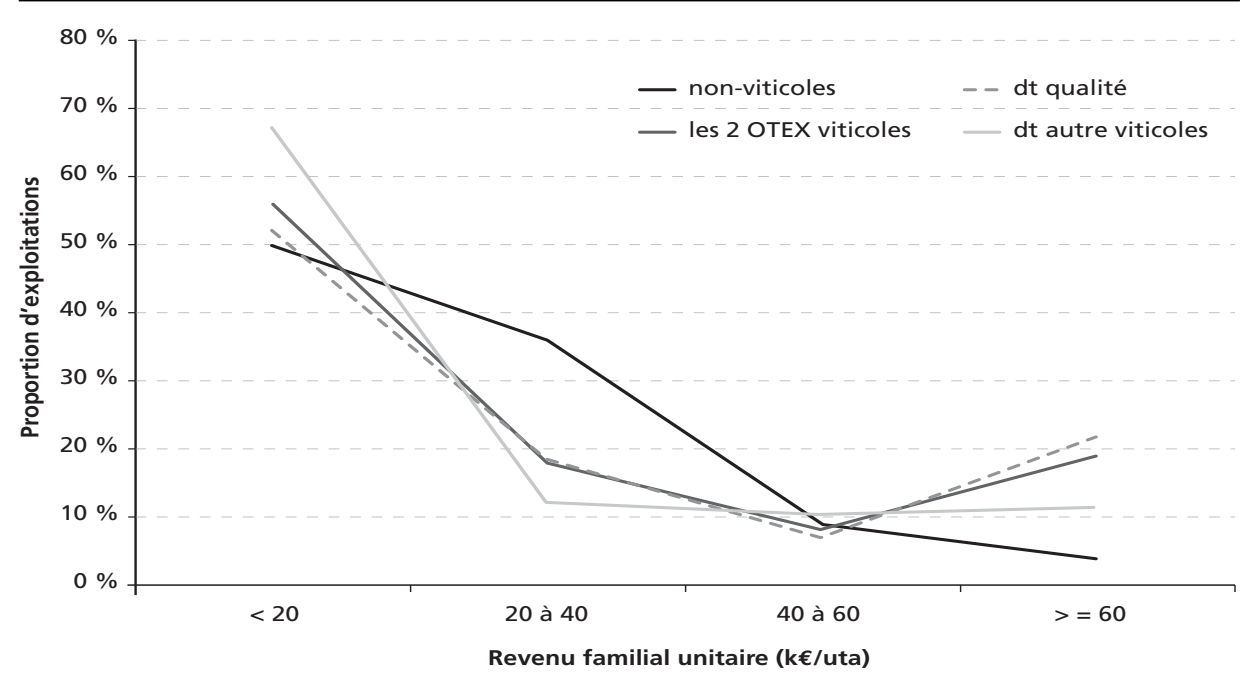

Source : RICA- échantillon constant-2005/2006/2007, traitement INRA-MOISA

plein n'atteint pas le niveau du SMIC (environ $20000 €$ ) ; dans l'OTEX « autre viticulture », ce sont les deux tiers des exploitations qui n'atteignent pas ce seuil.

Mais, à la différence des autres OTEX où elles ne sont que $4 \%$, il y a en viticulture une forte minorité (19\%) d'exploitations à revenu familial unitaire élevé, plus de $60 \mathrm{k} €$; valeur qui correspond approximativement au niveau de la productivité moyenne du travail dans l'économie française. On peut noter que la moitié de ces exploitations ne dépasse ce seuil qu'en rémunérant leurs salariés approximativement au niveau du SMIC, le revenu total étant inférieur à ce seuil.

Ainsi, les revenus des viticulteurs sont plus élevés en moyenne mais aussi plus hétérogènes que ceux des autres agriculteurs.

\section{Le rôle de la productivité du travail dans la dispersion des revenus}

À la recherche de facteurs explicatifs de l'hétérogénéité des revenus des viticulteurs, on peut considérer divers ratios : revenu par exploitation, par travailleur, par hectare, et part du revenu dans le montant de la production. Par ailleurs, la dispersion des revenus peut être mesurée par le coefficient de variation ${ }^{17}$ du revenu unitaire. Il est alors aisé de comparer plusieurs indicateurs du revenu : le revenu familial, comme précédemment ; le revenu total qui est la somme du revenu familial et du revenu des salariés ; la valeur ajoutée nette des amortissements.

Le montant de la production voit sa variabilité augmenter quand on le ramène à l'hectare de vigne, mais elle diminue quand on le calcule par unité de travail.

Des trois catégories de revenu, c'est le revenu familial qui a la plus grande variabilité, probablement en raison de la quasifixité de la rémunération unitaire des salariés. Les prélèvements « extérieurs » sur la valeur ajoutée (fermage, intérêts, taxes) accroissent peu la variabilité du solde. Notons que les variabilités sont très importantes puisque, par exemple pour le revenu familial, le coefficient de variation atteint ou dépasse 1,5 (c'est-à-dire, $150 \%$ ).

Enfin, c'est ramenée à l'hectare de vigne que la variabilité de la valeur ajoutée est maximale ; les ratios par exploita-

17. Le coefficient de variation est le quotient de l'écart-type par la moyenne. Ici, la moyenne des ratios ne doit pas être confondue avec le ratio moyen. 
FAITS ET CHIFFRES

Bernard DELORD

Tableau 2. Moyenne et variabilité de quelques ratios comptables des exploitations

\begin{tabular}{c|l|c|c|c|c}
\hline \multirow{2}{*}{$\begin{array}{c}\text { Dénominateurs } \\
\text { des ratios }\end{array}$} & \multirow{2}{*}{$\begin{array}{c}\text { Type } \\
\text { de statistiques }\end{array}$} & $\begin{array}{c}\text { montant de } \\
\text { la production }\end{array}$ & $\begin{array}{c}\text { valeur ajoutée } \\
\text { nette }\end{array}$ & revenu total & $\begin{array}{c}\text { revenu } \\
\text { familial }\end{array}$ \\
\cline { 3 - 6 } / exploitation & ratio moyen & 182,0 & 98,4 & 75,1 & 45,6 \\
\cline { 2 - 6 } & coeff. de var. & 1,11 & 1,30 & 1,39 & 1,69 \\
\hline \multirow{2}{*}{ / travailleur (uta) } & ratio moyen & 65,0 & 35,2 & 26,8 & 33,3 \\
\cline { 2 - 6 } & coeff. de var. & 0,62 & 0,91 & 1,04 & 1,91 \\
\hline \multirow{2}{*}{ / ha de vigne } & ratio moyen & 11,1 & 6,0 & 4,6 & 2,8 \\
\cline { 2 - 6 } & coeff. de var. & 1,37 & 1,59 & 1,66 & 1,82 \\
\hline \multirow{2}{*}{ / montant de } & ratio moyen & 1,00 & 0,54 & 0,41 & 0,25 \\
\cline { 2 - 6 } & coeff. de var. & 0,00 & 0,52 & 0,77 & 1,49 \\
\hline
\end{tabular}

Source : RICA- échantillon constant-2005/2006/2007, traitement INRA-MOISA

- Les ratios moyens sont en $k €$ (sauf quand le dénominateur est le montant de la production, ils sont alors sans dimension) ; les coefficients de variation sont sans dimension.

- Les deux cellules du ratio de revenu familial par travailleur concernent en fait le revenu familial par travailleur familial.

- Les deux questionnaires ayant un montant de production inférieur à 10 k€ sont exclus des deux dernières lignes.

tion, et surtout par UTA et par euro de production, présentent des écarts plus réduits. Il en est à peu près de même pour le revenu total.

Ces observations suggèrent l'existence d'un élément de variabilité important étranger à la dimension physique des exploitations. La vérification de cette hypothèse nous conduit d'abord à s'assurer du droit à écarter ce facteur.

\section{Dispersion des revenus et dimension des exploitations}

Par rapport aux autres OTEX, les relations entre la dimension des exploitations (et la productivité physique du travail) et le

Graphique 2. Niveau de revenu unitaire et dimension ou productivité physique
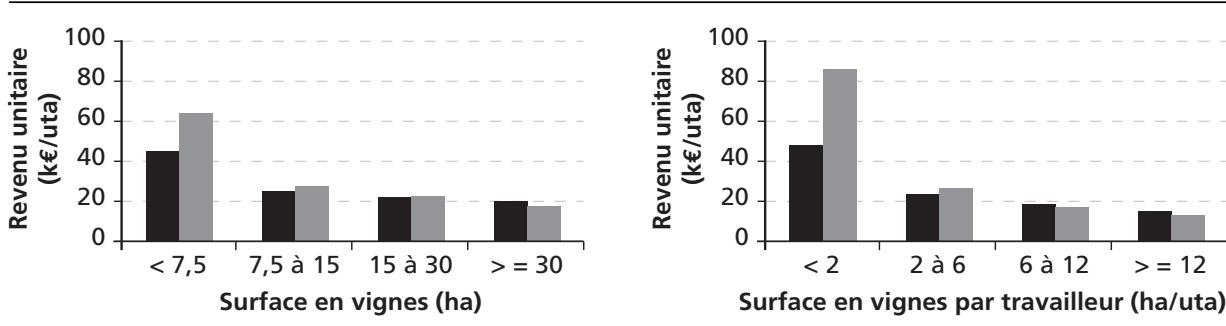

Surface en vignes par travailleur (ha/uta)
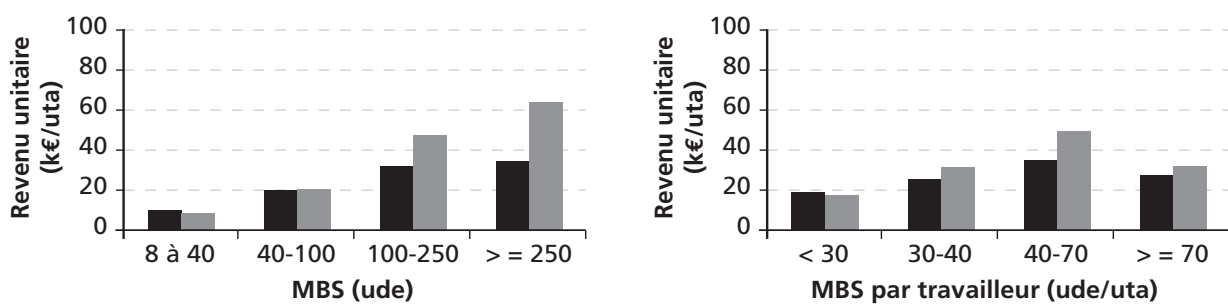

revenu total unitaire 


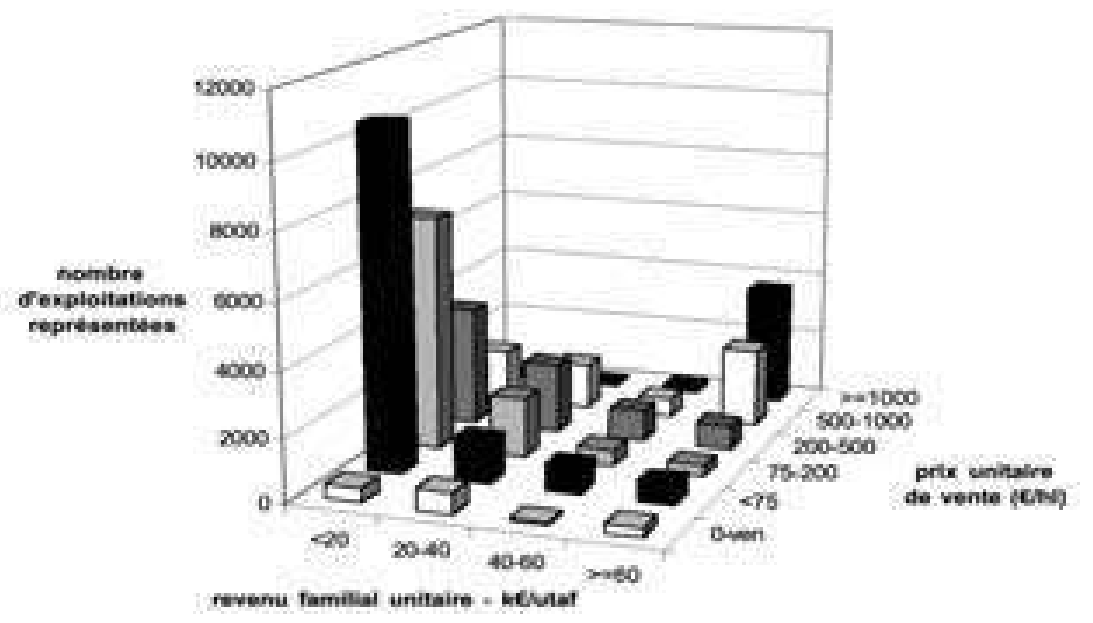

Source : RICA- échantillon constant-2005/2006/2007, traitement INRA-MOISA

niveau de revenu sont, en viticulture, assez singulières.

En considérant la surface en vigne, on constate qu'elle n'a, étonnamment, que peu d'influence sur le niveau de revenu par hectare pour les dimensions au-delà de 7,5 ha. Mais, c'est parmi les petites exploitations (moins de 7,5 ha), que l'on rencontre les revenus moyens par hectare les plus élevés. Inversement, parmi les exploitations moyennes ou grandes (plus de 7,5 ha), on trouve une large majorité de revenu familial unitaire très faible (moins de $20 \mathrm{k} € /$ UTA). Réciproquement, près des deux tiers des exploitations à haut revenu (plus de $60 \mathrm{k€} / \mathrm{UTA}$ ) ont moins de 7,5 ha de vigne. L'hypothèse classique de l'existence d'économies d'échelle semble ici invalidée.

Le ratio de la surface en vigne par UTA est une mesure de la productivité physique apparente du travail. Quand cette productivité est très faible (inférieure à 2 ha par UTA) le revenu unitaire moyen est élevé (48 k€/UTA). Pour les exploitations à niveau de productivité physique élevée ou très élevée (plus de 6 ha par UTA), les revenus unitaires sont le plus souvent très faibles (graphique 2). En forçant un peu le trait, on pourrait dire que plus la producti- vité physique apparente du travail croît et plus le revenu par travailleur diminue, c'est la tendance strictement inverse de celle constatée dans les autres OTEX.

Quant à la $\mathrm{MBS}^{18}$, les revenus augmentent avec elle. Parmi les très grandes exploitations (plus de $250 \mathrm{UDE}$ ), la proportion de revenus unitaires élevés (plus de $60 \mathrm{k} €$ ) atteint $40 \%$.

Si on mesure la productivité du travail à l'aide de la MBS par UTA, on observe bien une certaine tendance à l'augmentation des revenus quand cette productivité augmente, mais, pour les très hauts niveaux de productivité, la tendance semble s'inverser.

La prise en compte du revenu familial unitaire, au lieu du revenu total unitaire, ne fait qu'accentuer les tendances observées, ce qui est normal vu la fixité de la rémunération des salariés.

Au total, en viticulture, la dimension et la productivité physique du travail, quand elles sont mesurées par la surface en vigne,

18. Dans les OTEX non viticoles, la MBS est très nettement corrélée à la dimension physique ; dans la viticulture elle est fortement marquée par des valeurs des coefficients régionaux très différentes ; or, ces valeurs intègrent des prix moyens régionaux de vente unitaire du vin très différenciés, la MBS représente donc, en viticulture, plutôt une dimension économique. 
ne semblent pas ou très mal corrélées au revenu par travailleur. Le fait que la MBS y soit un peu mieux reliée semble indiquer qu'il faille porter attention à ce sur quoi reposent les différences régionales de coefficient de MBS (les consommations intermédiaires étant peu discriminantes), à savoir le prix de vente du vin.

\section{Le rôle primordial des prix unitaires}

La majorité du vin produit et des exploitations viticoles relèvent de la viticulture «d'appellation ». Les appellations sont des constructions juridiques, techniques, économiques et socioculturelles (Montaigne, 2001 ; Traversac, 1998, Laferté, 2006) ${ }^{19}$ qui différencient les produits. Elles sont un des fondements des écarts de prix

19. Montaigne E., Bruté de Rémur C. (2001). Métamorphose, diversité et reconquête. Agreste Languedoc-Roussillon. Le Bulletin, numéro spécial "Contributions à la connaissance de la viticulture régionale », juin, p. 77-84.

Traversac J.-B., Wavresky P., Moran W., PerrierCornet Ph. (1998). Devenir des appellations régionales de Bourgogne : une analyse par les systèmes de production. Montpellier, «Atelier d'économie viti-vinicole », 26-27 novembre, INRA.

Laferté G. (2006). La Bourgogne et ses vins. Image d'origine contrôlée. Belin, 320 p. de vente. Nous examinerons le lien des revenus avec les prix puis celui avec la localisation, avant de rechercher, une nouvelle fois, un effet de la dimension des exploitations, mais ici, au sein même de la relation entre le prix unitaire et le revenu.

\section{Le prix de vente unitaire du vin}

Parmi les données économiques a priori indépendantes de la surface, la plus immédiate est le prix unitaire de vente du vin ${ }^{20}$. Les différences de prix entre les exploitations sont considérables, surtout quand on les compare à celles des autres produits agricoles qui dépassent rarement quelques dizaines de pourcents. Pour un tiers des exploitations viticoles, le prix n'atteint pas $75 € / \mathrm{hl}$, mais pour $20 \%$ d'entre elles, il dépasse $500 € /$ hl.

La relation entre prix unitaire et niveau de revenu est très nette (graphique 3 ). À moins de 200 €/hl, l'immense majorité des exploi-

20. On privilégie le prix de vente unitaire du vin, malgré ses limites, par rapport au montant de la production par hectare de vigne car celui-ci tient compte du rendement (irrégulier selon les années), mêle des productions non viticoles (autres cultures, autres produits) et inclut les réévaluations de stocks, économiquement peu significatives.

\section{Graphique 4. Niveau de revenu unitaire et zones géographiques}

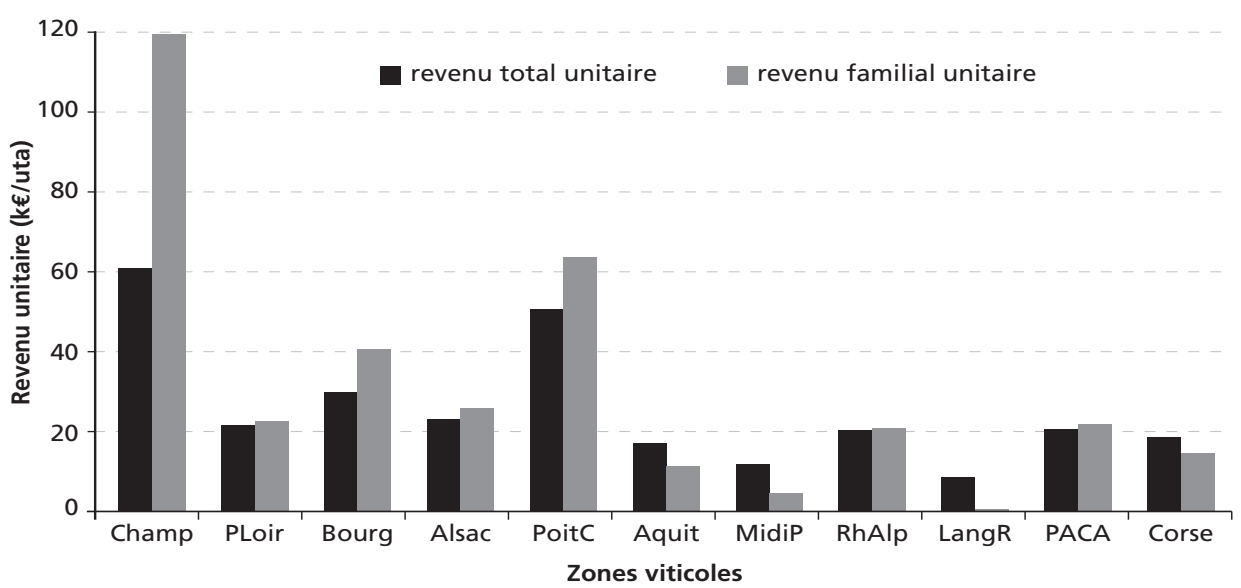

Source : RICA- échantillon constant-2005/2006/2007, traitement INRA-MOISA 
tations viticoles ont un revenu familial unitaire faible. Au-dessus de $500 € / \mathrm{hl}$, la majorité des revenus est moyenne ou élevée. Le graphique 3 montre nettement cet effet en « selle de cheval».

Mais, comment expliquer de telles différences dans les prix de vente unitaire du vin ? Le premier facteur est à rechercher dans la nature, la « qualité » du vin, c'est-àdire, principalement, la différenciation des vins par les appellations.

\section{Revenu et différenciation des vins}

Nous avons vu précédemment que le niveau de revenu variait nettement avec la présence ou l'absence d'appellation. Plus significative est la prise en compte de la localisation des exploitations, qui constitue une approche, très sommaire, de la différenciation des appellations.

Dans la zone Champagne et dans la région Poitou-Charentes, le revenu moyen unitaire par travailleur dépasse $50 \mathrm{k} €$. Alors qu'en Languedoc et Aquitaine, il est largement inférieur au niveau du SMIC $(20 \mathrm{k} €)$. Ici aussi et pour les mêmes raisons, la prise en compte du revenu familial accentue les écarts entre régions (graphique 4).
En considérant maintenant le type de commercialisation, on constate bien une différence dans les niveaux de revenu unitaire (graphique 5) : les embouteilleurs ont un revenu unitaire moyen supérieur à $30 \mathrm{k€}$ (supérieur à $50 \mathrm{k} €$ pour le revenu familial unitaire), alors que les non-embouteilleurs sont aux niveaux du SMIC. Ainsi, la mise en bouteille, souvent liée à la vente au détail, semble permettre aux viticulteurs, de s'approprier un niveau de valeur ajoutée plus élevé ${ }^{21}$. Cependant, l'écart est moins net que celui constaté selon le prix de vente unitaire $^{22}$, ce qui permet de supposer que, si la mise en bouteille par le viticulteur est un des

21. Le lien entre bouteille, prix et revenu est sans doute un peu plus complexe ; il conviendrait d'examiner les coûts et le travail supplémentaires engendrés par la mise en bouteille (et la vente directe) ; il faudrait aussi tester l'hypothèse que la mise en bouteille concerne préférentiellement des vins dont le prix de vente en vrac est plus élevé que celui des autres vins.

22. Du caractère moins étroit de cette relation peut, par exemple, témoigner le fait que, les 15322 exploitations représentées commercialisant de façon permanente en bouteille ont un revenu total unitaire moyen de $33,3 \mathrm{k} €$, alors que pour les 15343 exploitations ayant un prix de vente unitaire supérieur à $267 € / \mathrm{hl}$ ce même revenu est de $40 \mathrm{k} €$.

\section{Graphique 5. Niveau de revenu unitaire et type de commercialisation}

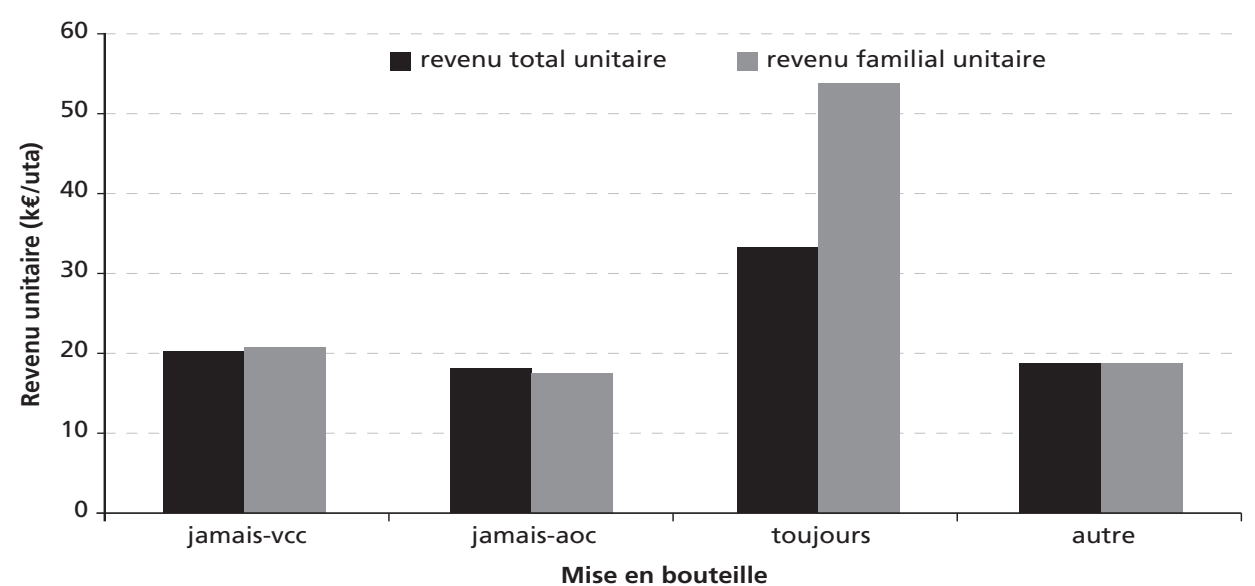

Source : RICA- échantillon constant-2005/2006/2007, traitement INRA-MOISA 


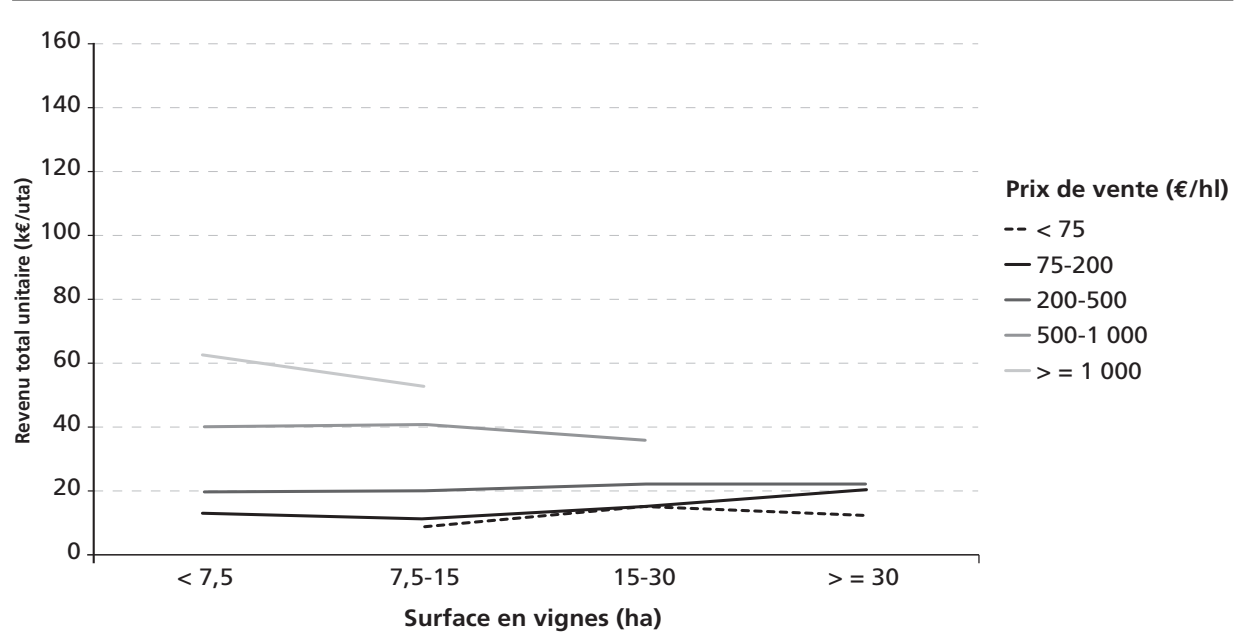

Source : RICA- échantillon constant-2005/2006/2007, traitement INRA-MOISA

éléments de différenciation des prix, elle ne peut, seule, la résumer ${ }^{23}$ (Vinifhlor, 2008) ${ }^{24}$.

\section{Revenu, prix de vente et dimension}

Ayant identifié le facteur principal de différenciation des revenus dans le niveau de prix unitaire de vente du vin, il est intéressant de croiser son effet avec celui de la dimension physique, principal facteur d'écarts de revenu dans les autres OTEX.

Pour les prix de vente bas (inférieurs à $200 € / \mathrm{hl})$, il semble y avoir une tendance à une certaine augmentation des revenus pour les surfaces les plus importantes ( $g r a$ phique 6), mais cette «compensation » ne concerne qu'un petit nombre d'exploitations ; la moitié des exploitations de plus de 30 ha de vigne sont, malgré tout, en dessous du niveau du SMIC.

23. Un travail portant sur qualité et profit dans la viticulture bourguignonne réalise une distinction comparable entre appellation, signe de qualité public, et comportement des entreprises, choix particuliers. Laporte C. (2000). Système d'information sur la qualité et profit: le cas des vins d'appellation d'origine contrôlée de Bourgogne. Thèse Université de Dijon.

24. Viniflhor (2008). Résultats 2006 : Observatoire des exploitations viticoles. Octobre, (consultable sur http://www.onivins.fr/pdfs/1217.pdf).
Entre 200 et $500 € / \mathrm{hl}$, l'effet de la taille en vigne sur le revenu est très modeste : on constate certes une petite augmentation de la proportion des revenus élevés parmi les grandes exploitations, mais la proportion de celles situées en dessous le niveau du SMIC reste forte (environ la moitié) et est quasiment indépendante de la dimension de la surface en vigne.

Au-delà de 500 €/hl, l'effet de la dimension est plus sensible, mais, comme l'indique le graphique 7 , cela semble beaucoup plus lié aux différences de rémunération entre salariés et non-salariés ${ }^{25}$ qu'à celles de la taille en vigne proprement dite ; pour les prix supérieurs à $1000 € / \mathrm{hl}$, l'effet est quasi nul ${ }^{26}$.

Au total, on vérifie que le facteur essentiel de la différenciation des revenus dans la viticulture est le niveau de prix de vente unitaire. L'importance de la dimension physique des exploitations ne permet qu'à un petit nombre d'entre elles, parmi celles vendant à prix uni-

25. En effet, la proportion de revenus totaux (incluant celui des salariés) unitaires élevés est, à la différence de celle des revenus familiaux unitaires élevés, quasiment indépendante de la dimension en vigne. 26. Il est possible que cet effet existe mais qu'il soit masqué par l'unicité de la classe au-delà de $60 \mathrm{k} €$, classe qui réunit $92 \%$ de l'ensemble des producteurs vendant leur vin à plus de $1000 € / \mathrm{hl}$. 


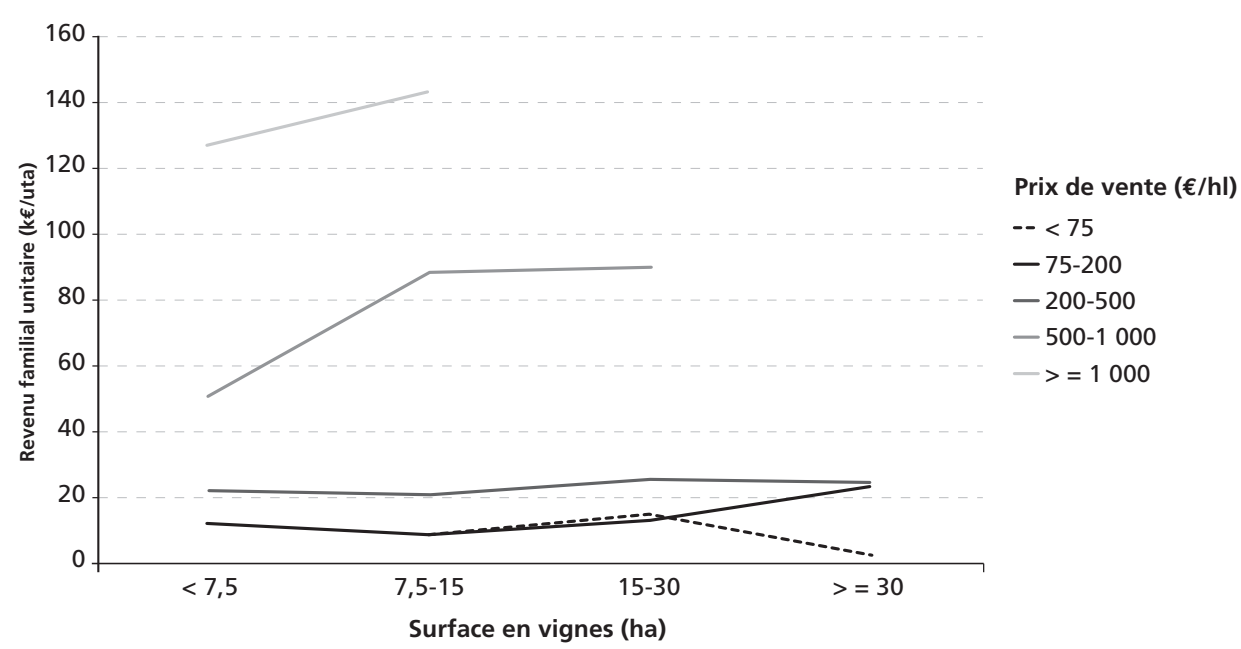

Source : RICA- échantillon constant-2005/2006/2007, traitement INRA-MOISA

taire bas, d' « échapper » aux bas revenus. Inversement, disposer d'une petite surface n'est qu'un handicap limité pour le revenu quand le prix de vente est moyen ou élevé27.

\section{Conclusion}

Le RICA français permet d'étudier certaines particularités du revenu des viticulteurs. Il faut néanmoins tenir compte de ses limites : entre autres, représentativité contrôlée seulement au niveau régional (ce qui s'adapte mal aux zones d'appellation) et impossibilité d'identifier les viticulteurs coopérateurs : seuls les vendeurs en bouteille sont distingués des vendeurs en vrac, parmi lesquels figurent les coopérateurs.

Quelle que soit la façon dont on les mesure, les revenus des viticulteurs ont une

27. De ce point de vue aussi, la viticulture est bien différente des autres orientations, puisque, par exemple en production laitière, si dans certaines régions (Savoies et Franche-Comté) le prix de vente du lait est supérieur à la moyenne nationale, cela n'empêche pas les exploitations d'y avoir un revenu moyen inférieur à la moyenne nationale car elles ont une dimension inférieure aux autres. Erhel A. (avec la participation de Rousset S., Wepierre A.-S., Piet L.) (2007). Les exploitations laitières : des situations régionales contrastées. Paris, Notes et études économiques, $\mathrm{n}^{\circ} 29$, décembre, p. 9-46. grande variabilité, plus grande que celle observée parmi les autres agriculteurs. À la différence des autres OTEX, cette variabilité n'est pas liée aux différences de taille (surface) des exploitations, pas plus qu'aux différences de productivité physique du travail que l'on explique généralement par les économies d'échelle. C'est le prix de vente unitaire du vin qui semble jouer le rôle principal dans la différenciation des niveaux de revenu des viticulteurs. Les écarts de prix entre exploitations sont considérables et se retrouvent pour l'essentiel dans les différences de revenu, les charges extérieures ne paraissant pas atténuer sensiblement ces écarts. Comme on pouvait s'y attendre au vu de la différenciation des produits et de la segmentation du marché, les différences de revenu sont assez nettement reliées au découpage géographique en régions. La vente en bouteille peut être considérée comme un élément alternatif de différenciation des revenus.

Il resterait à s'interroger sur l'origine de telles différences de prix de vente du vin. Il est certain que le système des appellations en est un facteur essentiel, mais il faudrait aussi distinguer les aspects collectifs et individuels de cette différenciation et particulièrement le rôle qu'y joue la vente en bouteille. 